\title{
Prediction of the secondary wake instability of a circular cylinder with direct numerical simulation
}

\author{
HONGYI JIANG ${ }^{1 \dagger}$, LIANG CHENG ${ }^{1,2}$, SCOTT DRAPER $^{1,3}$ and HONGWEI AN ${ }^{1}$ \\ ${ }^{1}$ School of Civil, Environmental and Mining Engineering, The University of Western Australia, 35 \\ Stirling Highway, Crawley, WA 6009, Australia \\ ${ }^{2}$ State Key Laboratory of Coastal and Offshore Engineering, Dalian University of Technology, \\ Dalian, 116024, China \\ ${ }^{3}$ Centre for Offshore Foundation Systems, The University of Western Australia, 35 Stirling \\ Highway, Crawley, WA 6009, Australia
}

\begin{abstract}
This paper presents an improved method for the prediction of the critical Reynolds number $R e_{c r}$ for the secondary wake instability of flow past a circular cylinder based on three-dimensional (3D) direct numerical simulations (DNS). It is also found that insufficient mesh resolution in the plane perpendicular to the cylinder axis is a major cause for the over-prediction of $\operatorname{Re}_{c r}(\sim 200)$ reported in the literature using 3D DNS. It is demonstrated that the use of an optimized computational domain and mesh resolution (10 spanwise mesh layers to resolve only a half of a pair of the 3D mode) can reduce the computational cost significantly and yet retain adequate accuracy. The most accurate $R e_{c r}$ predicted by the present DNS reduces to 190.74 , which is consistent with independent linear stability analysis results of $R e_{c r} \sim 190$. It is also demonstrated that 3D DNS is capable of accurately predicting the neutral curve for the secondary wake instability with a reasonable computational cost. The general conclusions drawn through a circular cylinder are expected to provide guidance on the prediction of $R e_{c r}$ and neutral instability curve for other cylindrical objects.
\end{abstract}

Key words: Direct numerical simulation; Circular cylinder; Secondary wake instability; Critical Reynolds number; Computational mesh; Computational cost

\footnotetext{
†Email address for correspondence: hongyijiang88@gmail.com
} 


\section{Introduction}

Instabilities in the wake of a long circular cylinder subjected to steady incoming flow have been the topic of extensive studies in the past few decades and have been used widely as a benchmarking problem for numerical sensitivity studies and the validation of numerical models. It is well known that the flow is governed by a single dimensionless parameter $R e$, which is defined based on the approaching flow velocity $U$, the cylinder diameter $D$ and the kinematic viscosity of the fluid $v$. Methods of investigation have included physical model tests, linear and non-linear stability analysis, and direct numerical simulations (DNS). Comprehensive reviews on investigations of flow with different methodologies can be found, for example, in Williamson (1996a, 1996b) and Posdziech and Grundmann (2001). Based on these investigations, it has been shown (e.g. Williamson 1996a) that for flow past a circular cylinder the flow structure in the wake undergoes a transition sequence of: (1) emergence of primary wake instability at $\operatorname{Re} \sim 49$, (2) onset of Mode A instability with large-scale vortex dislocations (i.e. Mode $A^{*}$ ) at $R e \sim 190$, (3) gradual transition from Mode $\mathrm{A}^{*}$ to Mode B over a range of $\operatorname{Re}$ from 230 to 250, and (4) development of increasingly disordered Mode B structures for $R e>260$.

Many early experimental studies have focused on identifying the critical Reynolds number $R e_{c r}$ at which the secondary instability Mode A* develops. The $R e_{c r}$ values identified by some experimental studies are: 150 by Roshko (1954) and Tritton (1959), 165 by Norberg (1994), 178 by Williamson $(1988,1989), 194$ by Williamson (1996b), and 205 by Miller and Williamson (1994). It was discovered that the low values of $R e_{c r}$ identified in early experimental studies were largely due to the "end effect" (Williamson, 1996b). Williamson (1996b) extended the $R e_{c r}$ value to 194 by eliminating end effects using non-mechanical end conditions. This value is fairly close to the $R e_{c r}$ values of $188.5( \pm 1.0), 190.2( \pm 0.02)$, and 190.5 predicted through linear stability analysis by Barkley and Henderson (1996), Posdziech and Grundmann (2001), and Rao et al. (2013), respectively.

In contrast to the linear stability analysis results, there has been considerable 
scatter in reported $R e_{c r}$ values obtained using non-linear three-dimensional (3D) DNS. Two different DNS approaches have been employed for the investigation of the flow. One is the spectral element approach that employs Fourier expansion to model spanwise variations in pressure and velocity, on the assumption of spanwise periodicity in the flow structure. The second is based on the conventional finite volume method (FVM) or finite element method (FEM) formulation that is routinely applied to flows involving complex geometries. An early 3D DNS study based on the spectral element method performed by Karniadakis and Triantafyllou (1992) found that the wake transitioned to 3D at $R e$ between 200 and 210. Persillon and Braza (1998) predicted an $R e_{c r}$ between 187 and 190 based on a FVM approach with a spanwise domain length of 2.25D. A recent 3D DNS study based on a FEM approach by Behara and Mittal (2010) with a span length of $10 D$ predicted an $\operatorname{Re}_{c r}$ of 200. Behara and Mittal (2010) also investigated the hysteresis effects at transition and found a hysteresis loop of $R e_{c r}$ between 197 and 200. The 3D DNS studies by Zhao et al. (2013) (FEM) and Tong et al. (2014) (FVM) both predicted $R e_{c r}$ values of above 200. Zhao et al. (2013) and Tong et al. (2014) attributed the over-prediction of $R e_{c r}$, compared to experimental results, to the absence of the "end effect" in the numerical simulations. However, given that the $R e_{c r}$ predicted by linear stability analysis without "end effect" is approximately 190 (e.g. Posdziech and Grundmann, 2001), the cause for the reported over-predictions of $R e_{c r}$ warrants further investigation.

In light of these earlier works, the primary aim of this study is to examine the ability of 3D DNS based on the FVM formulation to accurately predict $R e_{c r}$. It will be shown later on that the gap between $R e_{c r}$ predicted by the present DNS and those by experiments and linear stability analysis can be significantly narrowed with sufficient mesh resolution and computational domain size.

In an effort to reduce the high computational costs associated with achieving accurate prediction of $R e_{c r}$, a second aim of this paper is to explore strategies for minimizing the computational cost.

Since the numerical accuracy and computational cost for the prediction of $R e_{c r}$ by DNS has not been examined through a benchmarking study with sufficient accuracy, 
DNS has not been widely adopted for the prediction of $R e_{c r}$ for other bluff body flows and more general flows such as the flow over a backward-facing step. Alternatively, a common practice is to determine the $R e_{c r}$ value through linear stability analysis and to examine the non-linear flow beyond the secondary instability through DNS, although this may result in some inconsistence and some extra efforts for the investigation of a bluff body flow due to the use of two sets of numerical methods and computational meshes. Other than that, DNS serves as an alternative method for the prediction of $R e_{c r}$.

For other bluff body configurations, e.g. a square cylinder (Robichaux et al., 1999), an elliptic cylinder (Leontini et al., 2015), a circular ring (Sheard et al., 2003), two circular cylinders in staggered arrangements (Carmo et al., 2008), etc., and also for other more general flows such as the flow over a backward-facing step (Barkley et al., 2002) and the flow through a partially blocked channel (Griffith et al., 2007), it has been shown by linear stability analysis that the secondary instability of the flow is usually characterized by a 3D mode (e.g. Mode A) with a specific $R e_{c r}$ and a specific spanwise wavelength. Hence the general conclusions drawn from the present study through a circular cylinder are expected to provide guidance on the prediction of $R e_{c r}$ for other flows through an optimized DNS setup.

\section{Numerical model}

\subsection{Numerical method}

Numerical simulations have been carried out with OpenFOAM (www.openfoam.org) to solve the continuity and incompressible Navier-Stokes equations:

$$
\begin{aligned}
& \frac{\partial u_{i}}{\partial x_{i}}=0 \\
& \frac{\partial u_{i}}{\partial t}+u_{j} \frac{\partial u_{i}}{\partial x_{j}}=-\frac{1}{\rho} \frac{\partial p}{\partial x_{i}}+v \frac{\partial^{2} u_{i}}{\partial x_{j} \partial x_{j}}
\end{aligned}
$$

where $\left(x_{1}, x_{2}, x_{3}\right)=(x, y, z)$ are Cartesian coordinates, $u_{i}$ is the velocity component in 
the direction $x_{i}, t$ is time and $p$ is pressure. The same numerical approach used in Jiang et al. (2016a) is adopted here. Specifically, the FVM approach and the PISO (Pressure Implicit with Splitting of Operators) algorithm (Issa, 1986) are used to solve the equations. The convection, diffusion and time derivative terms are discretized using the fourth-order cubic scheme, the second-order linear scheme, and a blended scheme consisting of the second-order Crank-Nicolson scheme and a first-order Euler implicit scheme, respectively.

\subsection{Computational domain and mesh}

The computational domain and mesh are determined based on a thorough domain size and mesh resolution dependence study reported separately in Jiang et al. (2016a). The standard 3D mesh reported in Jiang et al. (2016a) is adopted in this study. Based on the standard 3D mesh, Jiang et al. (2016a) obtained DNS results of the wake transition of flow past a circular cylinder which are in good agreement with the experimental results reported in Williamson (1996b).

Some key parameters for the standard 3D mesh are specified as follows. A hexahedral computational domain of $50 D \times 40 D \times 12 D$ as shown in Fig. 1(a) is adopted. In the $x-y$ plane (the plane perpendicular to the cylinder span), the cylinder perimeter is equally discretized with 132 nodes. The radial size of the first layer of mesh next to the cylinder is $0.001 D$, which corresponds to a non-dimensional wall distance $y^{+}$of less than 0.05 for $R e \leq 250$. The cell expansion ratio in the whole domain is kept below 1.1. For the sake of wake structure visualization, a higher mesh resolution in the near wake is used by specifying a constant cell size along the $x$-direction for $x / D$ ranges from approximately 1.0 to 5.5 . A close-up view of the mesh in the $x$-y plane near the cylinder is shown in Fig. 1(b). The 3D mesh is formed by replicating the two-dimensional (2D) mesh along the $z$-axis, resulting in an identical mesh resolution in all planes perpendicular to the cylinder span. The cell size in the spanwise direction is $0.1 D$, which results in a total of 120 identical layers of mesh along the span. 
(a)

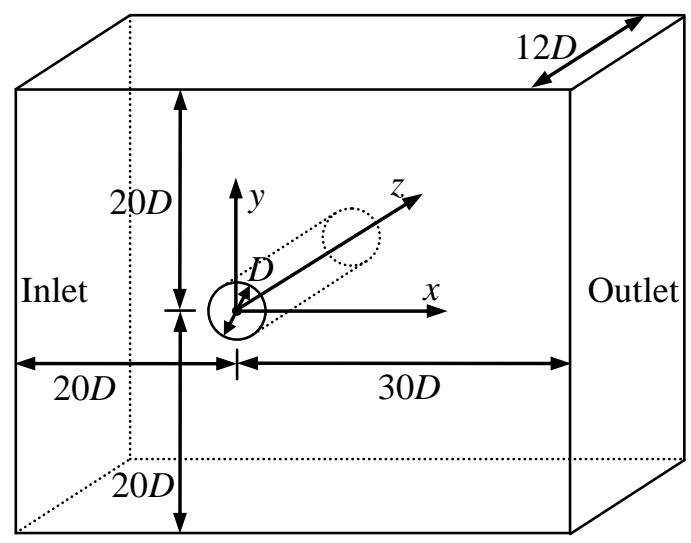

(b)

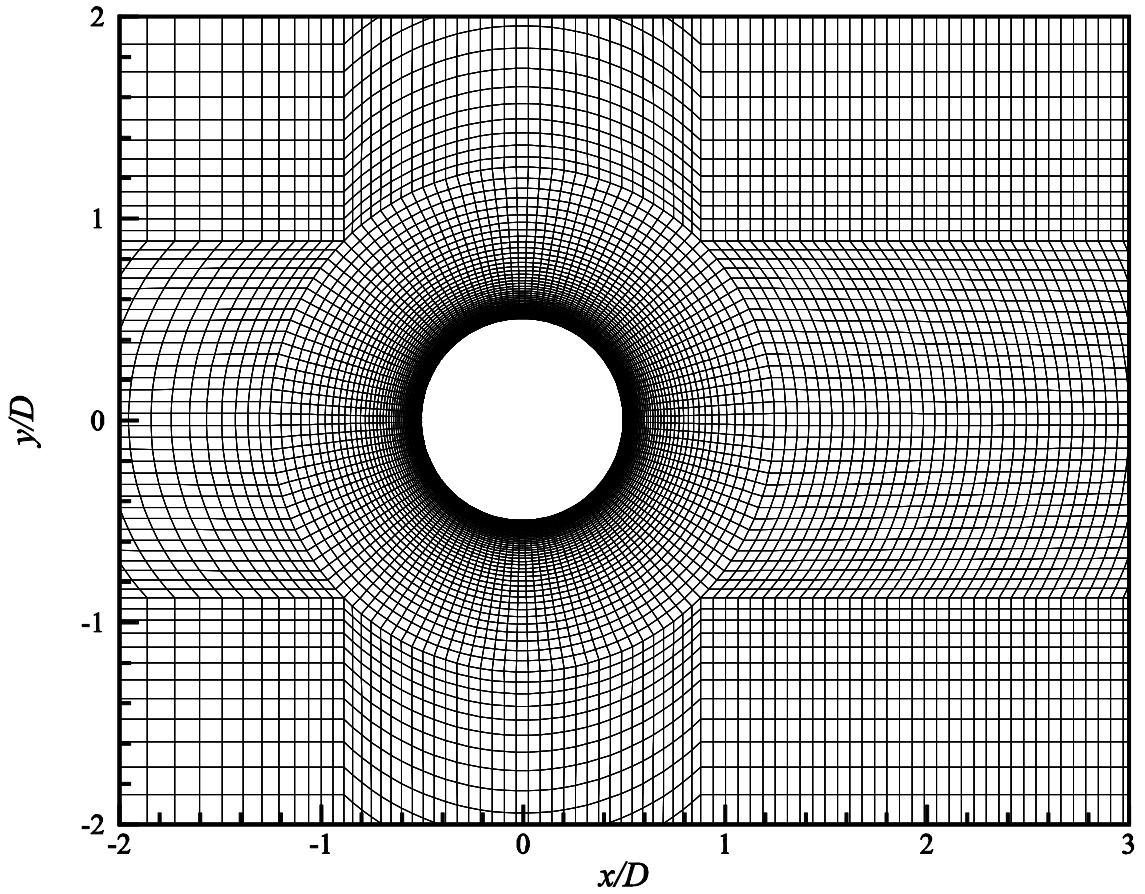

Fig. 1. (a) Schematic model of the computational domain, and (b) Close-up view of the mesh in the $x-y$ plane near the cylinder.

The boundary conditions adopted in the present study are the same as those used in Jiang et al. (2016a). At the inlet boundary, a uniform flow velocity $U$ is specified in the $x$-direction. At the outlet, the Neumann boundary condition (i.e. zero normal gradient) is applied for the velocity, and the pressure is specified as a reference value of zero. Symmetry boundary conditions are applied at the top and bottom boundaries, while periodic boundary conditions are employed at the two lateral boundaries perpendicular to the cylinder span. A non-slip boundary condition is applied on the cylinder surface.

The time step $\Delta t$ adopted for each case is based on the criterion that the Courant 
number $(\mathrm{Co})$ of all the cells in the computational domain is kept below 0.5 , where $C o$ is defined as:

$$
C o=|u| \Delta t / \Delta l
$$

where $|u|$ is the magnitude of the velocity through a cell, and $\Delta l$ is the cell size in the direction of the velocity. For example, this results in a non-dimensional time step (defined as $\Delta t^{*}=U \Delta t / D$ ) of $6.75 \times 10^{-3}$ at $\operatorname{Re}=250$. It is also checked that after reducing the time step by half (through keeping Co below 0.25 ) the variations of the hydrodynamic forces are within $1 \%$.

\section{Improved method for the prediction of $\boldsymbol{R} e_{c r}$}

In a previous 3D DNS study by Jiang et al. (2016a) by using the same numerical model as described in Section 2 with the standard 3D mesh, the numerical simulations were carried out with a Reynolds number interval of 1 , and the $R_{c r}$ value was determined by observing the emergence of the Mode $A^{*}$ instability and correspondingly a sudden drop of the Strouhal number $S t$ in the $S t-R e$ relationship. It was found in Jiang et al. (2016a) that the Mode A* instability and the sudden drop of St emerged at $R e=194$ but not at $R e=193$. Hence the $R e_{c r}$ value was between 193 and 194. In the present study, the $R e_{c r}$ value will be predicted with an improved method which allows for a higher precision of $R e_{c r}$ yet a lower computational cost.

\subsection{Improved precision of $R e_{c r}$}

To improve the precision of the prediction of $R e_{c r}$, the transient growth rate of the amplitude of spanwise velocity in the near wake is analysed. Fig. 2 shows two examples of the time-history of the spanwise velocity recorded at a sampling point $(x / D, y / D, z / D)=(3.0,0.5,6.0)$ in the near wake at $R e$ values slightly smaller and larger than $R e_{c r}$, respectively. The non-dimensional flow time $t^{*}$ in Fig. 2 is defined as $t^{*}=U t / D$. After a short initial fluctuation period, two distinct trends of the variation of spanwise velocity are observed: (i) For $R e<R e_{c r}$ (e.g. Fig. 2(a)), there is a convergence towards regular oscillations with a non-zero, but very small equilibrium 
amplitude $(\sim 0.01 U$ for $R e=193)$ and a well-defined period, and (ii) for $\operatorname{Re}>\operatorname{Re}_{c r}$ (e.g. Fig. 2(b)), there is a gradual development of oscillation amplitude in Stage 1, followed by a fully developed state of irregular oscillations with amplitudes close to $U$ (Stage 2). Fig. 3 shows the wake structures of the above two cases, visualized by the iso-surfaces of the normalized streamwise vorticity $\omega_{x}$ which is defined as:

$$
\omega_{x}=\left(\frac{\partial u_{z}}{\partial y}-\frac{\partial u_{y}}{\partial z}\right) \frac{D}{U}
$$

It is seen in Fig. 3(a) that for $R e=193\left(<R e_{c r}\right)$, the wake flow structure is represented by a small-amplitude stable state of Mode A structure (see Jiang et al., 2016b). For $R e$ $=194\left(>R e_{c r}\right)$, however, Stage 1 is characterized by the gradual development of the ordered Mode A structure (Fig. 3(b)), while the irregular and large-amplitude fluctuations of the spanwise velocity in Stage 2 are induced by the natural development of vortex dislocations (Fig. 3(c)). 
(a)

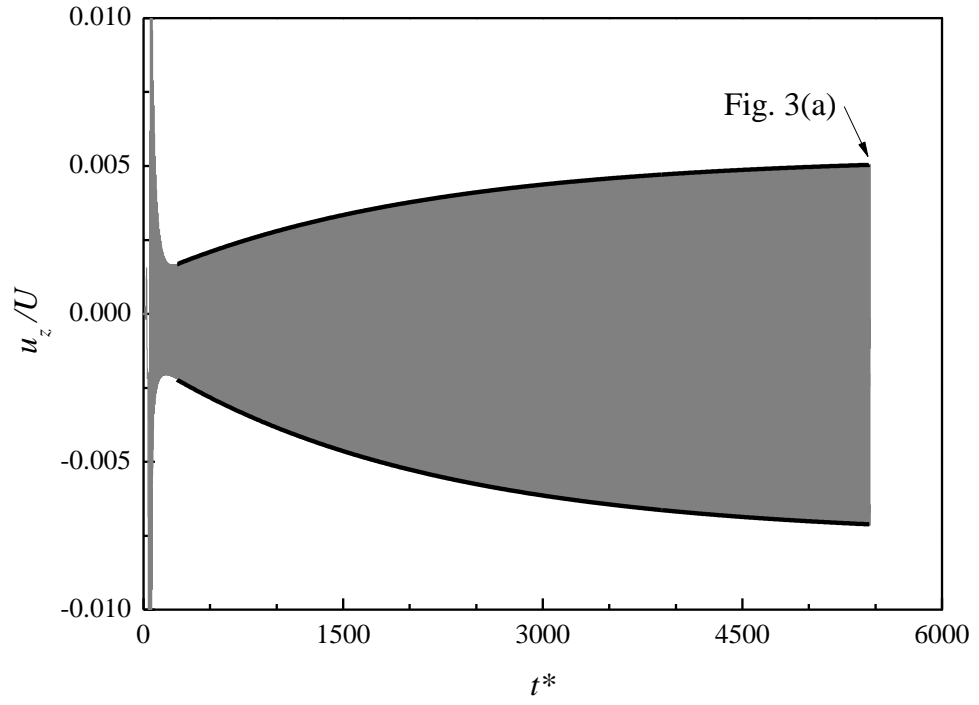

(b)

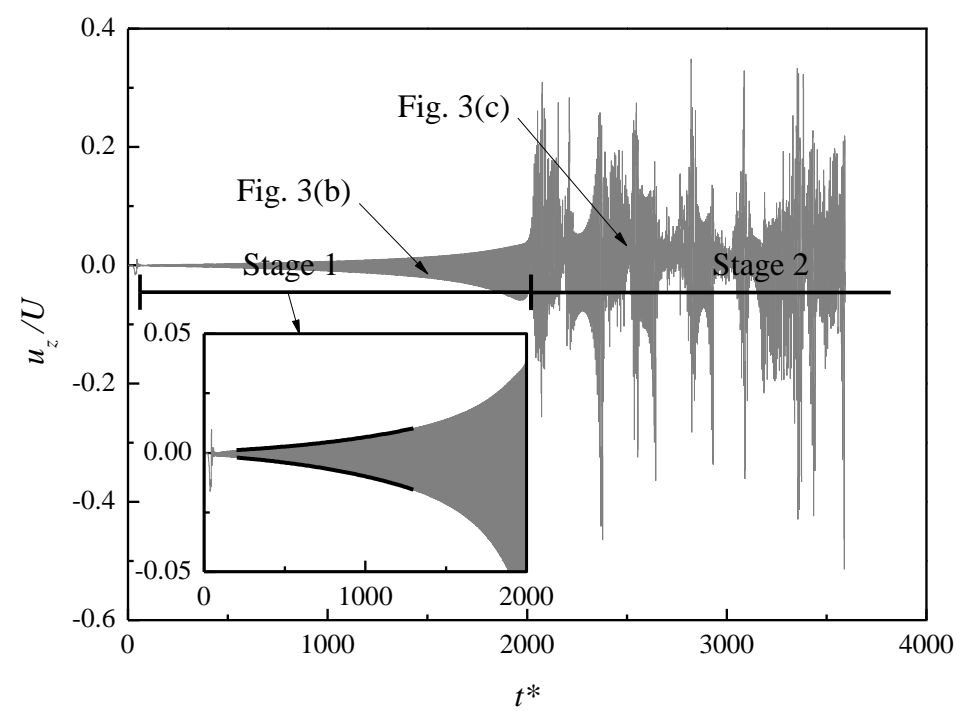

Fig. 2. Time-histories of the spanwise velocity sampled at $(x / D, y / D, z / D)=(3.0,0.5$, 6.0) for $R e$ slightly smaller and larger than $R e_{c r}$ : (a) $R e=193$, and (b) $R e=194$. 

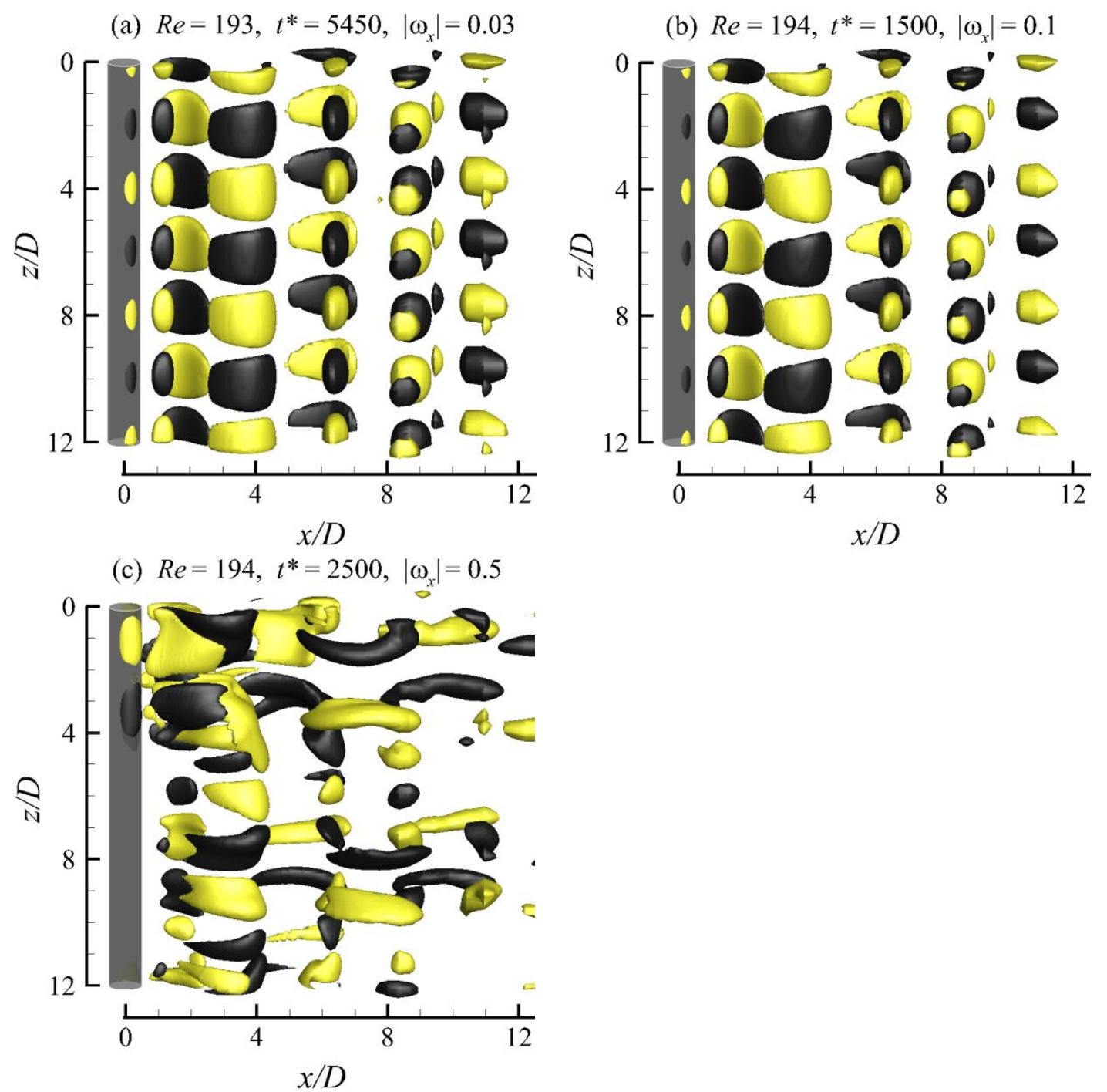

Fig. 3. Iso-surfaces of $\left|\omega_{x}\right|$ for $R e$ slightly smaller and larger than $R e_{c r}$. The iso-surfaces are shown in different $\left|\omega_{x}\right|$ values so as to display the main features of the wake structures. Dark grey and light yellow denote positive and negative values, respectively. The flow is from the left to the right past the cylinder on the left.

The gradual variations of the spanwise velocity amplitude with time, which represent the gradual convergence or development of the ordered Mode A flow, can be quantified by curve fitting upper and lower envelopes of the time-history of the spanwise velocity in the form of:

$u_{z} / U=A \exp \left(B t^{*}\right)+C$

where $A, B$ and $C$ are curve fitting coefficients. For example, the fitting results of the upper and lower envelopes marked by the solid curves in Fig. 2 are listed in Table 1. 
Although the upper and lower envelopes are asymmetric about the line of $u_{z} / U=0$ due to the use of the time-history of a point spanwise velocity sampled in the near wake (rather than integrated properties such as the lift coefficient), similar growth/convergence rates $B$ for the upper and lower envelopes are obtained. For the cases with $R e<R e_{c r}$, an exponential convergence of the spanwise velocity amplitude ( $B<0$, with the coefficient of determination $\mathrm{R}^{2}$ for the fitted curves very close to 1 ) is obtained. For $R e>R e_{c r}$, an exponential growth of the spanwise velocity amplitude $(B>$ 0 , with $\mathrm{R}^{2}$ also very close to 1 ) is obtained in the early development period where $u_{z} / U$ is within the order of 0.01 (see Fig. 2(b)). This suggests that $B=0$ corresponds to the neutral instability condition of $R e=R e_{c r}$.

Table 1. Exponential fitting results of the upper and lower envelopes marked by the solid curves in Fig. 2.

\begin{tabular}{lllll}
\hline Envelope & \multicolumn{2}{l}{ Coefficients for equation (3.2) } & \multirow{2}{*}{$\mathrm{R}^{2}$} \\
\cline { 2 - 4 } & $A$ & \multicolumn{2}{c}{$C$} & \\
\hline$R e=193$, upper envelope & $-4.124 \times 10^{-3}$ & $-4.881 \times 10^{-4}$ & $5.327 \times 10^{-3}$ & 0.99994 \\
$R e=193$, lower envelope & $6.008 \times 10^{-3}$ & $-4.849 \times 10^{-4}$ & $-7.543 \times 10^{-3}$ & 0.99994 \\
$R e=194$, upper envelope & $2.934 \times 10^{-3}$ & $1.137 \times 10^{-3}$ & $-2.545 \times 10^{-3}$ & 0.99994 \\
$R e=194$, lower envelope & $-3.776 \times 10^{-3}$ & $1.215 \times 10^{-3}$ & $2.844 \times 10^{-3}$ & 0.99993 \\
\hline
\end{tabular}

The cases with $R e$ close to $R e_{c r}$ are employed in the above analysis, since the growth/convergence periods can persist relatively longer and thus a more accurate $B$ value can be obtained. It is noted that the initial oscillations of each case as well as the non-linear irregularities for $R e>R e_{c r}$ (i.e. Stage 2) are not considered in the analysis. As shown in Fig. 4, a linear relationship between $B$ and $R e$ is obtained. The $R e_{c r}$ which corresponds to $B=0$ is 193.2 , which is consistent with the $R e_{c r}$ of between 193 and 194 obtained in Jiang et al. (2016a) but of a higher precision. 


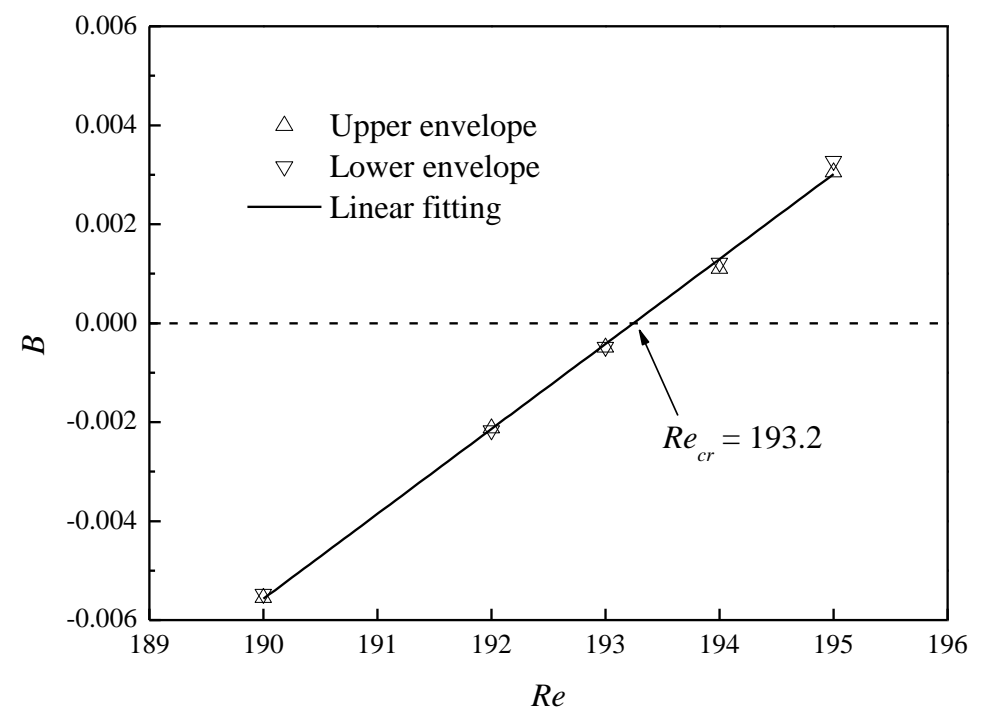

Fig. 4. Relationship between the growth/convergence rate $B$ and $R e$.

\subsection{Minimization of computational cost}

As shown in Fig. 3(a,b), in the gradual growth/convergence periods, the ordered Mode A streamwise vortex pairs are evenly distributed along the cylinder span width. In order to minimize the computational cost for the prediction of $R e_{c r}$, further $3 \mathrm{D}$ DNS are carried out by simulating only a half of a pair of the Mode A structure. Correspondingly, the mesh is modified from the standard 3D mesh:

(i) In order to accommodate only a half of a pair of the Mode A structure, the spanwise domain length $L_{z}$ is reduced to $2 D\left(=\lambda_{A} / 2\right.$, where $\lambda_{A}$ being the intrinsic spanwise wavelength of the Mode A structure which should be known a priori). In comparison with the use of $L_{z}=12 D\left(=3 \lambda_{A}\right)$ in Section 3.1, the computational cost by using $L_{z}=2 D$ is reduced significantly. Note that the intrinsic spanwise wavelength of the wake mode can be determined through either stability analysis (e.g. Barkley and Henderson, 1996) or the DNS method presented later on in Section 5 .

(ii) In order to simulate only a half of a pair of the Mode A structure, symmetry boundary conditions (rather than periodic boundary conditions) are applied at the lateral boundaries perpendicular to the cylinder span.

Based on the above modified mesh and the curve-fitting method introduced in 
Section 3.1, an $R e_{c r}$ of 193.3 is obtained. This $R e_{c r}$ value is of the same accuracy compared with using $L_{z}=12 \mathrm{D}$ but the computational cost is reduced significantly.

\section{Optimized mesh for the prediction of $R e_{c r}$}

The predicted $R e_{c r}$ of 193.2 by the present 3D DNS is closer to the experimental result of 194 (Williamson, 1996b) and those from linear stability analysis of $\sim 190$ (e.g. Posdziech and Grundmann, 2001) than those of $\sim 200$ by previous independent numerical simulations using non-linear 3D DNS. One possible reason for this improved prediction is that the mesh resolution used in the present study is better than those used in previous independent studies. However, the improved value of $R e_{c r}=$ 193.2 is still marginally higher that those predicted from linear stability analysis (of $\left.R e_{c r} \sim 190\right)$. Theoretically speaking, 3D DNS should be able to obtain an $R e_{c r}$ that is consistent with the values predicted by linear stability analysis. The question that remains is if we can further close the gap between the $R e_{c r}$ values predicted by non-linear 3D DNS and those by linear stability analysis.

\subsection{Evidence of mesh dependence}

It is suspected that the $R e_{c r}$ value based on the standard mesh may still be influenced by numerical diffusion associated with insufficient mesh resolution. Such insufficient mesh resolution is evidenced by the mesh dependence from a $2 \mathrm{D}$ mesh dependence study. According to the 2D mesh dependence study reported in Jiang et al. (2016a), among the mesh types investigated (e.g. doubling the domain size from the cylinder centre to each of the outer boundary, or doubling the amount of cells in both $x$ and $y$ directions), it was found that the overall mesh resolution in the $x-y$ plane is a major cause for the variation of the Strouhal number St which is defined as:

$S t=f_{L} D / U$

where $f_{L}$ is the frequency of the fluctuating lift force. To quantify the dependence of $S t$ on the mesh resolution in the $x-y$ plane, we define a mesh resolution factor $F_{m r}$ as: 
$F_{m r}=\left(\frac{\text { Total cell number of the current mesh }}{\text { Total cell number of the standard mesh }}\right)^{1 / 2}$

We then plot and curve-fit the variation of $S t$ with $F_{m r}$ for a set of 2D cases at $R e=$ 193 with different mesh resolutions (Fig. 5).

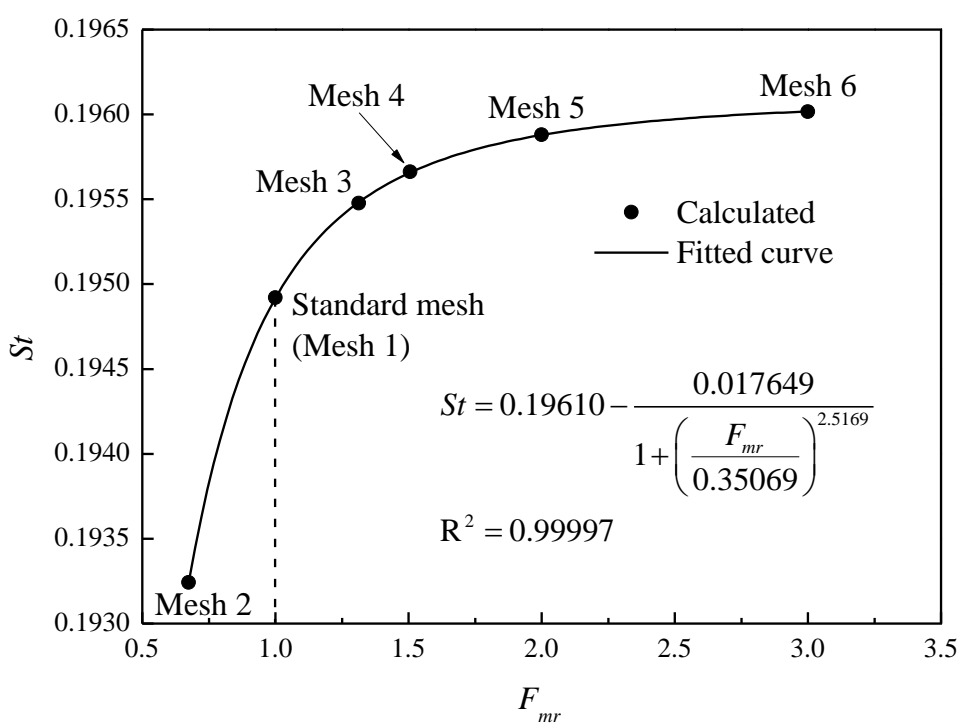

Fig. 5. Variation trend of $S t$ for a set of 2D cases with different mesh resolutions at $R e$ $=193$.

In Fig. 5, the case with $F_{m r}=1$ uses the standard mesh (in its 2D form) which was used in Section 3 (in its 3D form) to obtain $R e_{c r}=193.2$. Five more meshes, labelled Mesh 2 to Mesh 6, are adopted, with $F_{m r}$ being 0.674, 1.313, 1.507, 2.0 and 3.0, respectively. Based on the mesh resolution factor $F_{m r}$, each mesh has a cell number in both $x$ and $y$ directions equal to $F_{m r}$ times that for the standard mesh. Specifically, the number of nodes around the cylinder surface is $F_{m r}$ times that for the standard mesh, while the radial size of the first layer of mesh next to the cylinder is approximately $1 / F_{m r}$ times that for the standard mesh.

It is seen from Fig. 5 that the fitted $S t$ curve approaches an asymptotic value of 0.19610 as the mesh resolution is increased. The relative difference between the asymptotic $S t$ value and the one predicted using Mesh 6 is $0.60 \%$. Although the difference is quite small, it does suggest that the numerical results obtained with the 
standard mesh is still slightly influenced by the mesh resolution in the $x-y$ plane. For 3D simulations, it is reasonable to speculate that the predicted $R e_{c r}$ value may be also slightly affected by the mesh resolution in the $x-y$ plane if the standard 3D mesh is adopted.

\subsection{Cost-effective analysis of the mesh}

In addition to the mesh resolution in the $x-y$ plane, it is believed that other factors, including the mesh resolution in the spanwise direction, and the computational domain size in the $x-y$ plane, may also have influence on the prediction of $R e_{c r}$. It should be noted that $L_{z}(=2 D$ in this section unless otherwise stated) is determined based on the intrinsic spanwise wavelength of the emerging $3 \mathrm{D}$ wake structure and is not a factor for the convergence of $R e_{c r}$.

The influence of the mesh resolution in the spanwise direction on $R e_{c r}$ is examined first, since the increase in the spanwise cell size $(\Delta z)$ will significantly reduce the total amount of cells in the computational domain. The same curve-fitting method as described in Section 3.1 is used to determine $R e_{c r}$. Fig. 6(a) shows the dependence of $\operatorname{Re}_{c r}$ on $\Delta z$. It is found that insufficient mesh resolutions (i.e. large cell sizes) in the spanwise direction lead to over-predictions of $R e_{c r}$, which is mainly due to numerical diffusion associated with insufficient resolutions. Based on a well-fitted exponential curve, a limit value of $R e_{c r}=193.23$ is obtained with decrease of $\Delta z$. With respect to the limit value, the use of $\Delta z=0.1 D$ and $\Delta z=0.2 D$ lead to small relative errors of approximately $0.05 \%$ and $0.16 \%$, respectively. With further increase of $\Delta z$ to $0.4 D$, a much larger relative error of approximately $0.89 \%$ is obtained. To compromise between the numerical accuracy and the computational cost, further calculations will be carried out with $\Delta z=0.2 D$ (i.e. use 20 spanwise mesh layers to resolve a pair of the Mode A structure).

The dependence of $R e_{c r}$ on the computational domain size in the $x-y$ plane is examined next. This part of the investigation is further decoupled by examining the influence of the domain sizes from the cylinder centre to the inlet, outlet, and side (top 
and bottom) boundaries separately, and the results are shown in Fig. 6(b). It is seen in Fig. 6(b) that a lower $R e_{c r}$ value is obtained when the domain size from the cylinder centre to either side of the outer boundary is restricted to $10 D$, especially for a restricted domain size from the cylinder centre to the inlet. This is attributed to the blockage effect of the flow which results in a higher effective $R e$ and thus a lower $R e_{c r}$. When the domain size from the cylinder centre to either side of the outer boundary increases to $20 D$, the blockage effect becomes much weaker and the relative errors of the predicted $R e_{c r}$ with respect to that obtained with a domain size of $160 D$ are all less than $0.3 \%$. In particular, the relatively errors become negligible $(<0.1 \%)$ when the domain size from the cylinder centre to the outlet boundary is larger than $20 D$.

(a)

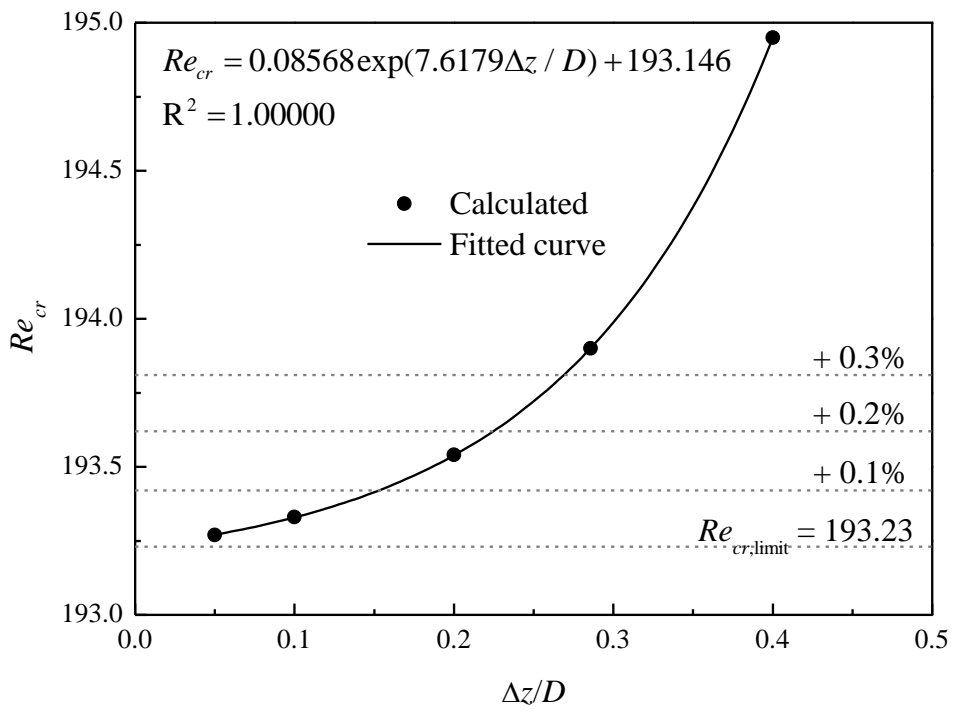

(b)

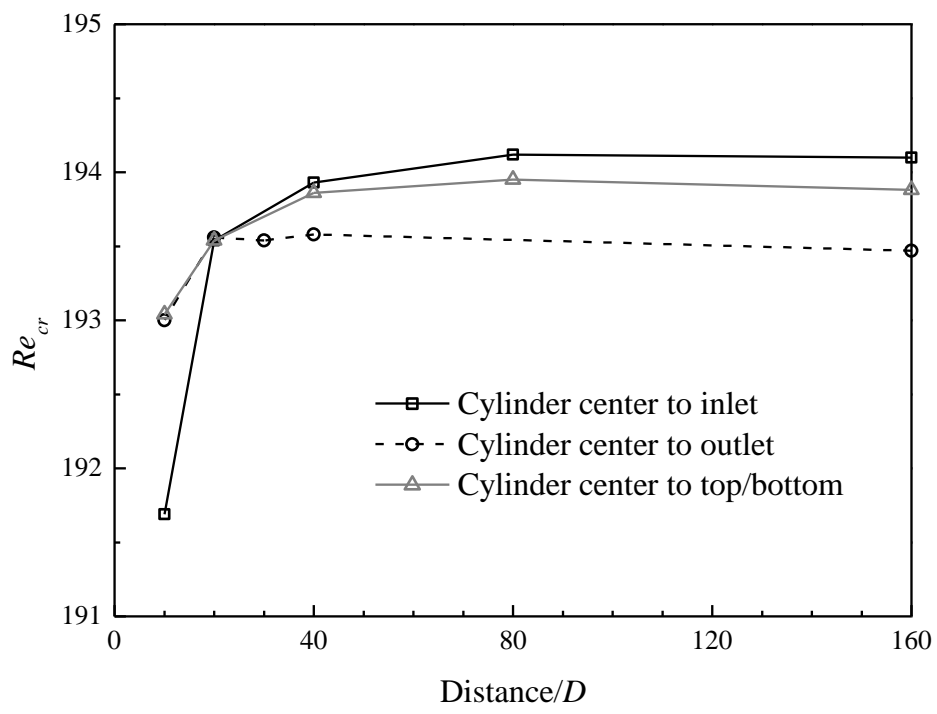


(c)

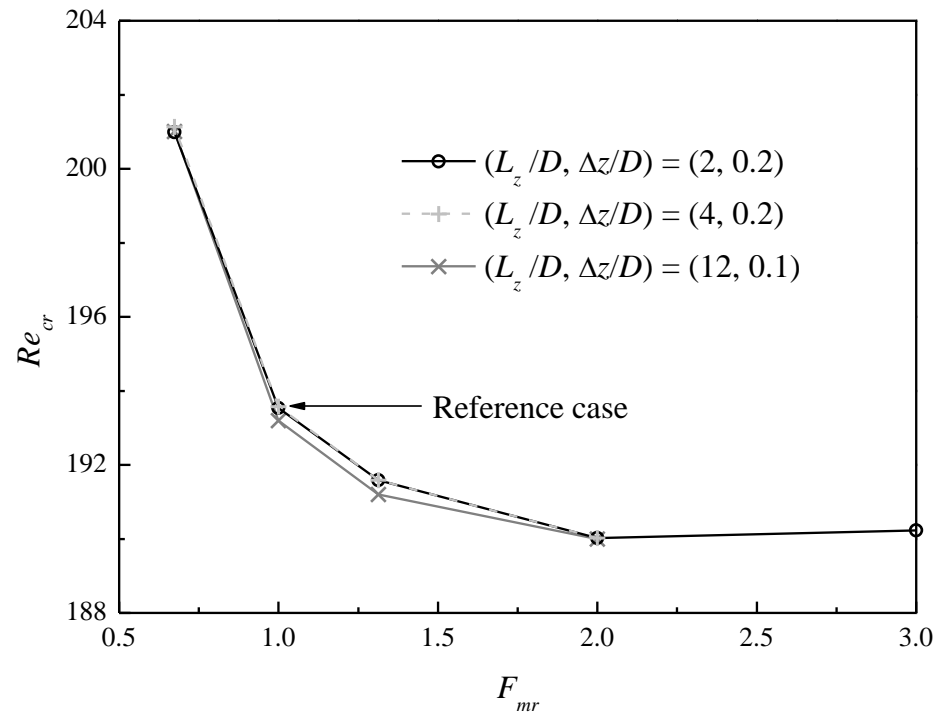

Fig. 6. Mesh dependence of the $\operatorname{Re}_{c r}$ : (a) on the cell size in the spanwise direction, (b) on the computational domain size in the $x-y$ plane, and (c) on the mesh resolution in the $x-y$ plane.

The dependence of $R e_{c r}$ on the mesh resolution in the $x-y$ plane is examined with $\left(L_{z} / D, \Delta z / D\right)=(2,0.2),(4,0.2)$, and $(12,0.1)$, and the results are shown in Fig. 6(c). As expected, the $R e_{c r}$ values predicted with $\left(L_{z} / D, \Delta z / D\right)=(2,0.2)$ and $(4,0.2)$ are almost identical, while slightly lower $R e_{c r}$ values (still in very close agreement) are observed with $\left(L_{z} / D, \Delta z / D\right)=(12,0.1)$ due to the decrease of $\Delta z / D$ from 0.2 to 0.1 .

The computational costs for the cases with $\left(L_{z} / D, \Delta z / D\right)=(2,0.2)$ and $(12,0.1)$ are listed in Table 2 and are reported by the number of core hours (hoursxprocessors) being used for the calculation of 500 non-dimensional flow time (which is normally required for the determination of the growth/convergence rate $B$ ). Note that with the increase of the total cell number in the computational domain (e.g. with the increase of $F_{m r}$ or $L_{z}$ ), more processors are used for the calculations in order to reduce the wall-clock time so that the calculations can be accomplished within a few days. The total number of core hours for each case is normalized with the computational cost for the reference case of $\left(F_{m r}, L_{z} / D, \Delta z / D\right)=(1,2,0.2)$ and is given in the bracket of Table 2. 
Table 2. Computational costs for the cases with $\left(L_{z} / D, \Delta z / D\right)=(2,0.2)$ and $(12,0.1)$. The total number of core hours for each case is normalized with the cost for the reference case of $\left(F_{m r}, L_{z} / D, \Delta z / D\right)=(1,2,0.2)$ and is given in the bracket.

\begin{tabular}{lll}
\hline$F_{m r}$ & \multicolumn{2}{l}{ Computational time (hours $\times$ processors $)$} \\
\cline { 2 - 3 } & $\left(L_{z} / D, \Delta z / D\right)=(2,0.2)$ & $\left(L_{z} / D, \Delta z / D\right)=(12,0.1)$ \\
\hline 0.674 & $0.96 \times 24(0.29)$ & $6.30 \times 120(9.43)$ \\
1 & $3.34 \times 24($ Reference case $)$ & $19.75 \times 256(63.07)$ \\
1.313 & $8.66 \times 24(2.59)$ & $31.99 \times 256(102.16)$ \\
2 & $32.92 \times 96(39.43)$ & $153.77 \times 360(690.58)$ \\
3 & $184.68 \times 144(331.76)$ & Not attempted \\
\hline
\end{tabular}

It is seen in Table 2 that the computational cost for the reference case (3.34 hours on a 24-processor Cray XC40 supercomputer node) is quite acceptable. Compared with the reference case with $F_{m r}=1$, the computational cost is increased by a factor of 39.43 by using $F_{m r}=2$, which is not very practical if a large amount of cases are simulated. It should be noted that when $F_{m r}$ increases from 1 to 2 , the computational cost will at least increase by a factor of $2^{3}$, due to the increase of the total cell number in the computational domain and the reduction of the time step to maintain a constant Courant number. Apart from that, more computational time may be required for a finer mesh due to an increased number of iterations for numerical convergence, as well as the non-linear scaling effect for parallel computation with more processors.

It is also seen in Table 2 that much higher computational costs are required by using $\left(L_{z} / D, \Delta z / D\right)=(12,0.1)$ with respect to $\left(L_{z} / D, \Delta z / D\right)=(2,0.2)$, which is mainly due to the significant increase of mesh layers in the spanwise direction. Due to impractical computational costs, the case with $F_{m r}=3$ is not attempted.

Based on $\left(L_{z} / D, \Delta z / D\right)=(2,0.2)$, it is seen in Fig. 6(c) that the relative error of the predicted $R e_{c r}$ by using $F_{m r}=2$ and 3 is only approximately $0.1 \%$. However, the relative error increases to $1.7 \%$ for the reference case with $F_{m r}=1$, and further increases to $5.7 \%$ when an even coarser mesh with $F_{m r}=0.674$ is adopted. Similar to 
the over-prediction of $R e_{c r}$ due to insufficient mesh resolutions in the spanwise direction as shown in Fig. 6(a), it is seen in Fig. 6(c) that insufficient mesh resolutions in the $x-y$ plane also lead to over-predictions of $R e_{c r}$, which is also caused by numerical diffusion associated with insufficient resolutions.

It is also seen in Fig. 6(c) that all three cases with $F_{m r}=0.674$ predict $R e_{c r}$ values of approximately 201, which are in line with the $R e_{c r}$ values of approximately 200 predicted by previous independent 3D DNS (e.g. Karniadakis and Triantafyllou, 1992; Behara and Mittal, 2010; Zhao et al., 2013; Tong et al., 2014). This case demonstrates that inadequate mesh resolutions in the $x-y$ plane are primarily responsible for over-predictions of $R e_{c r}$ of approximately 200. Note that unlike linear stability analysis, previous 3D DNS would normally adopt relatively large $L_{z}$ of more than $10 D$, and perform the simulation for a rather long flow time to obtain a fully developed flow for the examination of the occurrence of the secondary instability and thus the determination of $R e_{c r}$. Hence a relatively coarse mesh in the $x-y$ plane would normally be adopted to obtain sensible numerical results within a reasonable time frame. For example, the numbers of nodes around the cylinder surface, which serve as a key parameter for the mesh resolution in the $x-y$ plane, were 80,80 , and 100 in Behara and Mittal (2010), Zhao et al. (2013), and Tong et al. (2014), respectively. In comparison, the numbers of nodes around the cylinder surface for $F_{m r}=0.674$ and 1 in the present study are 88 and 132, respectively. It is seen that the mesh resolutions for the above-mentioned previous DNS studies were more in line with the present mesh with $F_{m r}=0.674$ than the mesh with $F_{m r}=1$. This suggests that the over-prediction of $R e_{c r}$ is indeed mainly due to an inadequacy of the mesh resolution in the $x-y$ plane.

Recall that for the mesh dependence study results shown in Fig. 6, the reference case has a computational domain size of $(20 D+30 D) \times(20 D+20 D) \times 2 D$, with $\Delta z=$ $0.2 D$ and $F_{m r}=1$, and the predicted $R e_{c r}$ is 193.54. Table 3 lists the relative errors of the predicted $R e_{c r}$ for the reference case with respect to the most accurate $R e_{c r}$ values obtained from each part of the mesh dependence study. With a simple summation of all the relative errors listed in Table 3 (of $+1.47 \%$ ), the most accurate $R e_{c r}$ after 
considering all the factors listed in Table 3 is 190.74, which is very close to the linear stability analysis results reported in the literature.

Table 3. Relative errors of the predicted $R e_{c r}$ for the reference case with respect to the most accurate $R e_{c r}$ values obtained from each part of the mesh dependence study.

\begin{tabular}{lllll}
\hline Parameter & $\begin{array}{l}\text { Reference } \\
\text { case }\end{array}$ & $\begin{array}{l}\text { Most accurate } \\
\text { case }\end{array}$ & $\begin{array}{l}\text { Most accurate } \\
R e_{c r}\end{array}$ & $\begin{array}{l}\text { Relative error } \\
\text { of } R e_{c r}\end{array}$ \\
\hline$\Delta z$ & $0.2 D$ & Limit condition & 193.23 & $+0.16 \%$ \\
Cylinder centre & $20 D$ & $160 D$ & 194.10 & $-0.29 \%$ \\
to inlet & & & & \\
Cylinder centre & $20 D$ & $160 D$ & 193.88 & $-0.18 \%$ \\
to top/bottom & & & & \\
Cylinder centre & $30 D$ & $160 D$ & 193.47 & $+0.04 \%$ \\
to outlet & & & & $+1.74 \%$ \\
$F_{m r}$ & 1 & 3 & 190.23 & \\
\hline
\end{tabular}

\section{Prediction of the neutral instability curve}

To extend on the prediction of $R e_{c r}$, the neutral curve for the secondary wake instability is also attempted with 3D DNS. As a compromise between the computational cost and accuracy for the prediction of $R e_{c r}$, the simulations are carried out with $F_{m r}=1$ and a computational domain size in the $x-y$ plane of $(20 D+$ $30 D) \times(20 D+20 D)$. The spanwise domain length $L_{z} / D$ varies from 1.35 to 3.4 , which corresponds to spanwise wavelengths $\lambda / D$ of 2.7 to 6.8 due to the use of symmetry boundary conditions at the lateral boundaries (if a half of a pair of the $3 \mathrm{D}$ mode is resolved). 10 identical layers of mesh are used in the spanwise direction to resolve a half of a pair of the Mode A structure. This is consistent with the use of $\Delta z / D=0.2$ for $L_{z} / D=2$ in Section 4.2 .

The neutral curve for the secondary instability predicted by the present 3D DNS is shown in Fig. 7, together with the neutral curves predicted by Barkley and 
Henderson (1996) and Posdziech and Grundmann (2001) through linear stability analysis. According to Barkley and Henderson (1996), flow past a circular cylinder is linearly unstable to $3 \mathrm{D}$ perturbations only if the wavelength of perturbations falls in the area enclosed by the neutral curve at corresponding $R e$ values, and the points on the neutral curve correspond to the neutral state of the flow where perturbations neither grow nor decay. It is seen in Fig. 7 that the $R e_{c r}$ values predicted by the present DNS are marginally to the right of the neutral curves predicted by Barkley and Henderson (1996) and Posdziech and Grundmann (2001). As suggested in Table 3, the slight over-prediction of $R e_{c r}$ is mainly due to an insufficient mesh resolution in the $x-y$ plane (with $F_{m r}=1$ ).

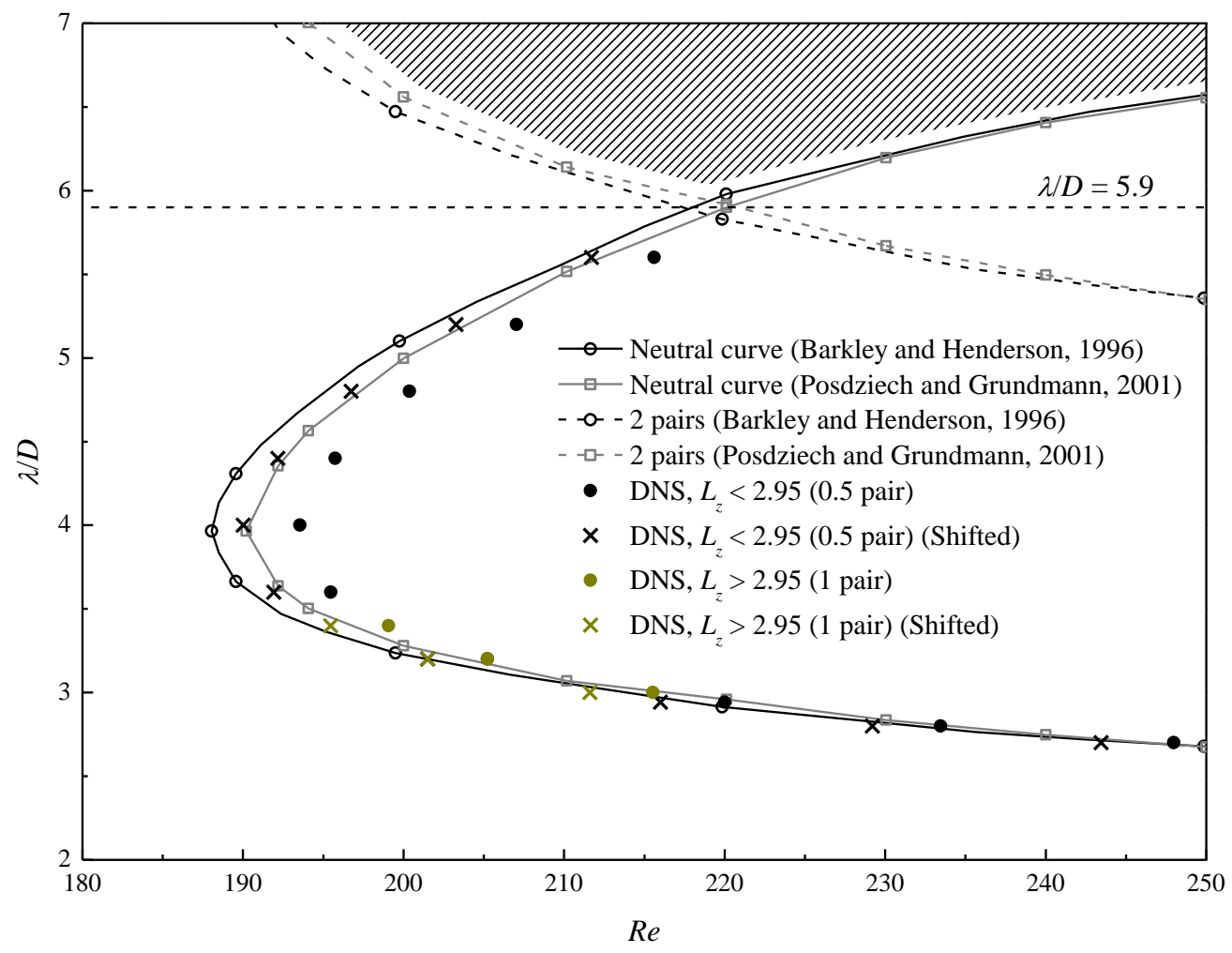

Fig. 7. Neutral curve for the secondary wake instability.

The accuracy of the neutral curve predicted through DNS is improved by taking into account the effect of mesh resolution in the $x-y$ plane. It is seen in Fig. 6(c) that when $F_{m r}$ increases from 1 to 2 , the predicted $R e_{c r}$ at the left tip of the neutral curve (i.e. with $L_{z} / D=2$ ) decreases by $1.82 \%$ and generally reaches convergence. To take 
into account the effect of mesh resolution in the $x-y$ plane, all of the $R e_{c r}$ values predicted by the present DNS are reduced by $1.82 \%$, and it is seen in Fig. 7 that the shifted $R e_{c r}$ values are in very good agreement with the neutral curves predicted by Barkley and Henderson (1996) and Posdziech and Grundmann (2001) through stability analysis.

Similarly, for other bluff body flows and other more general flows involving a secondary wake instability characterized by a 3D wake mode with a specific $R e_{c r}$ and a specific spanwise wavelength (such as those mentioned in Section 1), the neutral curve for the secondary instability can be obtained by DNS based on the following two steps:

(i) Plot the neutral curve based on a mesh that is less refined in the $x-y$ plane (e.g. the $F_{m r}=1$ mesh in the present study) as a compromise between the computational cost and numerical accuracy. Note that the use of a computational domain size of $20 D$ from the cylinder centre to each side of the outer boundary and 10 spanwise mesh layers to resolve a half of a pair of the 3D wake mode would generally result in fairly accurate predictions.

(ii) Improve the accuracy of the neutral curve by taking into account the influence of mesh resolution in the $x-y$ plane. This can be estimated by calculating the $R e_{c r}$ at the left tip of the neutral curve with a mesh that is refined in the $x-y$ plane (e.g. the $F_{m r}=2$ mesh in the present study).

It is worth noting that the neutral curves obtained through DNS and linear stability analysis are slightly different. For example, as shown in Fig. 7, the part of the neutral curve with $\lambda / D>5.9$ cannot be reproduced by the DNS approach. It is seen in Fig. 7 that the neutral curves for the development of one and two pairs of the Mode A wake structure intersect at $\lambda / D \sim 5.9$. For $\lambda / D>5.9$, the secondary instability first occurs on the neutral curve for two pairs of Mode A (and $\lambda / D$ should be reduced by half) rather than the neutral curve for one pair of Mode A (Fig. 7), and for Re in between the neutral curves for one and two pairs of Mode A (the shadow area) the flow is already in a $3 \mathrm{D}$ state.

In the present DNS, a half of a pair of Mode A is observed for $L_{z} / D<2.95$ (e.g. 
Fig. 8(a)), which corresponds to $\lambda / D=2 L_{z} / D<5.9$. In contrast, one pair of Mode A is observed for $L_{z} / D=3,3.2$, and 3.4 (e.g. Fig. 8(b)), for which $\lambda$ equals to $L_{z}$. This suggests that $\lambda$ is capped at $\sim 5.9$ as shown in Fig. 7. On the other hand, the lower branch of the neutral curve can be well reproduced by DNS to much higher $R e$ values (Fig. 7). It is thus concluded that the DNS approach actually predicts the onset points for an unforced secondary instability (with spontaneously generated wake structures) under different span lengths, which are in line with the neutral curves forced to one or more pairs of wake structures but truncated at their first intersection.

(a)

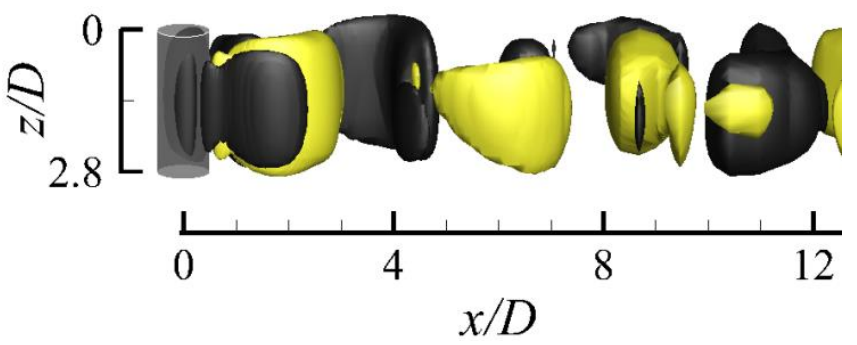

(b)

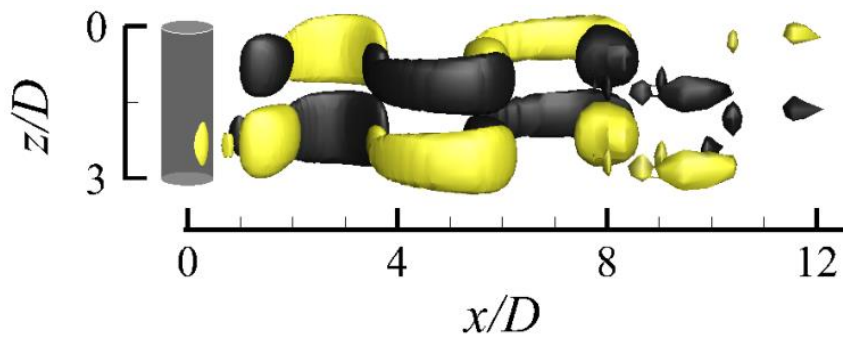

Fig. 8. Iso-surfaces of $\left|\omega_{x}\right|=0.01$ at $R e=217$ (slightly larger than $R e_{c r}$ ) and $t^{*}=500$ with (a) $L_{z}=2.8$ (development of a half of a pair of Mode A), and (b) $L_{z}=3.0$ (development of one pair of Mode A). Dark grey and light yellow denote positive and negative values, respectively. The flow is from the left to the right past the cylinder on the left.

\section{Conclusions}

This paper presents a systematic investigation on the prediction of the critical Reynolds number $R e_{c r}$ for the secondary wake instability of flow past a circular cylinder based on three-dimensional (3D) direct numerical simulations (DNS). It is found that the predicted $R e_{c r}$ value is sensitive to numerical mesh resolution and 
computational domain size. Insufficient mesh resolution either along the cylinder axis or in the plane perpendicular to the cylinder axis (the $x-y$ plane) leads to an over-prediction of $R e_{c r}$, which is mainly due to numerical diffusion associated with insufficient mesh resolutions. On the other hand, insufficient domain size from the cylinder centre to either side of the outer boundary leads to an under-prediction of $R e_{c r}$, which is attributed to the blockage effect of the flow which results in a higher effective $R e$ and thus a lower $R e_{c r}$. It is found that insufficient mesh resolution in the $x-y$ plane is a major cause for the over-prediction of $\operatorname{Re}_{c r}(\sim 200)$ reported in the literature using 3D DNS.

It is also demonstrated that the use of an optimized computational domain and mesh resolution (10 spanwise mesh layers to resolve only a half of a pair of the 3D mode) can reduce the computational cost significantly and yet retain adequate accuracy. The most accurate $R e_{c r}$ predicted by the present DNS reduces to 190.74 , which is consistent with independent linear stability analysis results of $R_{e_{c r}} \sim 190$.

To the authors' knowledge, it is demonstrated for the first time that the neutral curve for the secondary wake instability can be predicted through 3D DNS with a reasonable computational cost, and the results are in close agreement with the neutral curves determined by previous linear stability analysis. Furthermore, it is important to note that the DNS approach actually predicts the onset points for an unforced secondary instability (with spontaneously generated wake structures) under different span lengths, which are in line with the neutral curves forced to one or more pairs of wake structures but truncated at their first intersection.

The general conclusions drawn from the present study through a circular cylinder are expected to provide guidance on the prediction of $R e_{c r}$ and neutral instability curve for other cylindrical objects.

\section{Acknowledgments}

This work was supported by resources provided by the Pawsey Supercomputing Centre with funding from the Australian Government and the Government of Western 
Australia. The first author would like to acknowledge the support from the Australian Government and the University of Western Australia by providing IPRS and APA scholarships for a doctoral degree, as well as the Australia-China Natural Gas Technology Postgraduate Research Scholarships from the Australian and Western Australian Governments, the North West Shelf Joint Venture Partners, and the Western Australian Energy Research Alliance. The fourth author would like to acknowledge the support from the Australian Research Council through Discovery Early Career Research Award (DE150100428).

\section{References}

Barkley, D., Henderson, R.D., 1996. Three-dimensional Floquet stability analysis of the wake of a circular cylinder. Journal of Fluid Mechanics 322, 215-241.

Barkley, D., Gomes, M.G.M., Henderson, R.D., 2002. Three-dimensional instability in flow over a backward-facing step. Journal of Fluid Mechanics 473, 167-190.

Behara, S., Mittal, S., 2010. Wake transition in flow past a circular cylinder. Physics of Fluids 22, 114104.

Carmo, B.S., Sherwin, S.J., Bearman, P.W., Willden, R.H.J., 2008. Wake transition in the flow around two circular cylinders in staggered arrangements. Journal of Fluid Mechanics 597, $1-29$.

Griffith, M.D., Thompson, M.C., Leweke, T., Hourigan, K., Anderson, W.P., 2007. Wake behaviour and instability of flow through a partially blocked channel. Journal of Fluid Mechanics 582, 319-340.

Issa, R.I., 1986. Solution of implicitly discretized fluid flow equations by operator-splitting. Journal of Computational Physics 62, 40-65.

Jiang, H., Cheng, L., Draper, S., An, H., Tong, F., 2016a. Three-dimensional direct numerical simulation of wake transitions of a circular cylinder. Journal of Fluid Mechanics 801, $353-391$

Jiang, H., Cheng, L., Tong, F., Draper, S., An, H., 2016b. Stable state of Mode A for flow past a circular cylinder. Physics of Fluids 28, 104103. 
Karniadakis, G.E., Triantafyllou, G.S., 1992. Three-dimensional dynamics and transition to turbulence in the wake of bluff objects. Journal of Fluid Mechanics 238, 1-30.

Leontini, J.S., Lo Jacono, D., Thompson, M.C., 2015. Stability analysis of the elliptic cylinder wake. Journal of Fluid Mechanics 763, 302-321.

Miller, G.D., Williamson, C.H.K., 1994. Control of three-dimensional phase dynamics in a cylinder wake. Experiments in fluids 18, 26-35.

Norberg, C., 1994. An experimental investigation of the flow around a circular cylinder: influence of aspect ratio. Journal of Fluid Mechanics 258, 287-316.

OpenFOAM. Available from www.openfoam.org.

Persillon, H., Braza, M., 1998. Physical analysis of the transition to turbulence in the wake of a circular cylinder by three-dimensional Navier-Stokes simulation. Journal of Fluid Mechanics $365,23-88$.

Posdziech, O., Grundmann, R., 2001. Numerical simulation of the flow around an infinitely long circular cylinder in the transition regime. Theoretical and Computational Fluid Dynamics 15, $121-141$

Rao, A., Thompson, M.C., Leweke, T., Hourigan, K., 2013. The flow past a circular cylinder translating at different heights above a wall. Journal of Fluids and Structures 41, 9-21.

Robichaux, J., Balachandar, S., Vanka, S.P., 1999. Three-dimensional Floquet instability of the wake of square cylinder. Physics of Fluids 11, 560-578.

Roshko, A., 1954. On the development of turbulent wakes from vortex streets. NACA report 1191.

Sheard, G.J., Thompson, M.C., Hourigan, K., 2003. From spheres to circular cylinders: the stability and flow structures of bluff ring wakes. Journal of Fluid Mechanics 492, 147-180.

Tong, F., Cheng, L., Zhao, M., Zhou, T., Chen, X., 2014. The vortex shedding around four circular cylinders in an in-line square configuration. Physics of Fluids 26, 024112.

Tritton, D.J., 1959. Experiments on the flow past a circular cylinder at low Reynolds numbers. Journal of Fluid Mechanics 6, 547-567.

Williamson, C.H.K., 1988. The existence of two stages in the transition to three-dimensionality of a cylinder wake. Physics of Fluids 31, 3165-3168.

Williamson, C.H.K., 1989. Oblique and parallel modes of vortex shedding in the wake of a circular cylinder at low Reynolds numbers. Journal of Fluid Mechanics 206, 579-627. 
Williamson, C.H.K., 1996a. Vortex dynamics in the cylinder wake. Annual Review of Fluid Mechanics 28, 477-539.

Williamson, C.H.K., 1996b. Three-dimensional wake transition. Journal of Fluid Mechanics 328, $345-407$.

Zhao, M., Thapa, J., Cheng, L., Zhou, T., 2013. Three-dimensional transition of vortex shedding flow around a circular cylinder at right and oblique attacks. Physics of Fluids 25, 014105. 